\title{
The accuracy of volume flow measurements derived from pulsed wave Doppler: a study in the complex setting of forearm vascular access for hemodialysis
}

\author{
Abigail Swillens \\ Koen Van Canneyt \\ Patrick Segers \\ IBiTech-bioMMeda \\ Ghent University, Ghent, Belgium \\ Email: abigail.swillens@ugent.be
}

\author{
Lasse Lovstakken \\ Department of Circulation and Medical Imaging \\ NTNU, Trondheim, Norway \\ St. Olavs University Hospital, Trondheim, Norway
}

\begin{abstract}
Purpose: Maturation of an arterio-venous fistula (AVF) frequently fails, with low postoperative fistula flow as a prognostic marker for this event. As pulsed wave Doppler (PWD) is commonly used to assess volume flow, we studied the accuracy of this measurement in the setting of a radiocephalic AVF. Methods: As in-vivo validation of fistula flow measurements is cumbersome, we performed simulations, integrating computational fluid dynamics with an ultrasound (US) simulator. Flow in the arm was calculated, based on a patient-specific model of the arm vasculature pre and post AVF creation. Next, raw ultrasound signals were simulated, from which the Doppler spectra were calculated in both a proximal (brachial) and a distal (radial) location. Results: The velocity component in the direction of the US beam, in a centred, small, sample volume, can be captured accurately using PWD spectrum mean-tracking. However, deriving flow rate from these measurements is prone to errors: (i) the angle-correction which is influenced by the radial velocity components in the complex flow field; (ii) the largest error is introduced due to a lack of knowledge on the spatial flow profile.
\end{abstract}

\section{INTRODUCTION}

In Europe, more than half-a-million patients with end-stage renal disease (ESRD) are treated with hemodialysis and this population still increases annually by approximately $5-8 \%$. To make hemodialysis possible, a well-functioning vascular access is needed to facilitate high blood flow and to allow repeated cannulation over time. The preferred vascular access is created by surgically connecting an artery and a vein in the arm, i.e. an arteriovenous fistula or AVF. Ideally, the AVF matures, resulting in the required flow increase and vascular remodelling to allow for dialysis. Despite the extensive preoperative examinations, non-maturation occurs in 23 to $46 \%$ of the cases [1].

Direct post-operative flow measurements can be linked, in an early-stage, with the non-maturation in case of a too low fistula flow and with distal ischemia and cardiac failure in case of a too high fistula flow [2]. Nowadays, one of the most widely available flow measuring methods is based on pulse wave Doppler (PWD), where PWD provides velocity information that is multiplied with an estimate of the cross- sectional area of the vessel to obtain volumetric flow. Although PWD is a well-established method to measure flow velocities, it has some intrinsic limitations. The most important one is the 1D-nature of the measurement, since PWD only captures the velocity component in the direction of the US-beam. The translation of this 1D velocity measurement to the full crosssectional velocity profile, in order to estimate the flow rate, requires a great number of assumptions. These uncertainties involved in the flow rate calculation are expected to increase significantly when a disturbed and complex flow field is introduced, as one may expect post AVF-creation. So, despite the fact that the PWD flow estimation is non-invasive and used on large scale in daily routine, the reproducibility, intra- and inter-observer variability, and the accuracy remain unclear.

In this study, we want to assess the accuracy of the flow rate as measured by ultrasound (US) compared to the actual flow rate present in the setting of AVF. To ensure that we have gold standard information on the true velocity field measured by PWD, we will follow an approach based on multiphysics simulations, integrating computational fluid dynamics (CFD) with an ultrasound simulator. As we demonstrated before, this modelling technique allows to simulate synthetic but realistic ultrasound images, based on a known but complex 3D-flow situation [3]. As such, we will produce synthetic PWD spectra to derive flow rates, which can then be compared to the ground truth values known from CFD. For this analysis, two patientspecific geometries of one AVF patient, based on pre- and postoperative MR-images of the arm vasculature, will be used.

\section{Methods}

\section{A. Multiphysics simulations}

1) Flow simulations in a patient-specific model of AVF using CFD: Two sets of high-resolution MR angiography images were acquired from the same patient (51 years old male), one set pre-operatively and one 15 weeks after AVF creation. From these MR data sets, both a pre-operative and a post-operative 3D-model were reconstructed using appropriate software (http://www.vmtk.org). The pre-operative 


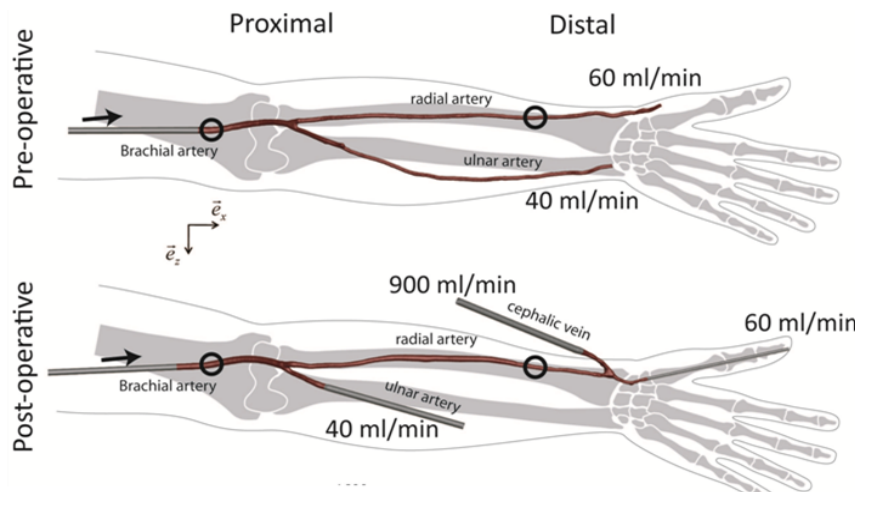

Fig. 1. Preoperative and postoperative simulation setup.

model consists of the brachial artery, which bifurcates into the ulnar and the radial artery (fig. 1, top). The post-operative model additionally includes the arterio-venous anastomosis and the distal cephalic vein (fig. 1, bottom). A computational mesh of the models was constructed for the CFD-simulations using pyFormex (http://pyformex.org), resulting in a structured hexahedral grid of 1.4 million cells for the pre-operative case and 2.2 million cells for the post-operative case.

The CFD-simulations further require that appropriate boundary conditions are applied at the in- and outlets of the geometry. As such, a parabolic velocity profile was implemented as inlet boundary condition at the proximal brachial artery, as derived from pre- and post-operative MRI Q-flow acquisition from the selected patient. Traction-free conditions were applied at the outlets. Blood was modelled as an incompressible Newtonian fluid with a density of $1050 \mathrm{~kg} / \mathrm{m}^{3}$ and a viscosity of $3.5 \mathrm{mPa} \cdot \mathrm{s}$. Ansys Fluent 12 (ANSYS inc., Canonsburg, PA, USA) was used to solve the Navier-Stokes equations with a finite volume method.

2) Ultrasound simulations: We used the Field II software [4], [5] to simulate the radiofrequent (RF) signals originating from the AVF blood flow fields. Field II represents blood as an ensemble of random point scatterers on which the US waves reflect. As such, realistic Doppler spectra were obtained by moving the point scatterers during the simulated scanning procedure according to the AVF flow fields obtained from CFD. We implemented a realistic linear array transducer for peripheral vascular applications, and RF-signals were obtained by firing 8000 and 22000 sinusoidal pressure pulses during the cardiac cycle, for the pre-operative and post-operative case respectively ( $\mathrm{f} O=5 \mathrm{MHz}$, pulse length $=2.5$ pulse periods). RFdata were simulated at 2 locations (o in fig.1): (i) the brachial artery, $5 \mathrm{~cm}$ proximal to the radial-ulnar bifurcation (proximal location), and (ii) the radial artery, $5 \mathrm{~cm}$ proximal to the anastomosis (distal location). Finally, the Doppler sonograms were produced by choosing an appropriate depth and size of the sample volume, and applying Fourier-analysis to the simulated RF-signals. Using a mean frequency estimator, the velocity in the US-beam direction, $v_{P W D}$, was derived from the Doppler spectra. To reduce the variance of the spectrum estimates, the final velocity curve was obtained by averaging power spectra from several individual range samples within the sample volume. For more background on the ultrasound simulator and the applied multiphysics simulation approach, we refer to [3].

\section{B. Volume flow calculation based on PWD spectra}

The instantaneous volume flow, $\mathrm{Q}(\mathrm{t})$, through a well-defined cross-section, A, can be calculated as the integration of the spatial velocity profile over the area:

$$
Q(t)=\int_{A}(\vec{v}(t) \cdot \vec{n}) d A=\int_{A} v_{n}(t) d A
$$

with $\vec{v}(t)$ the 3D-velocity vector in a certain point of the crosssection, $\vec{n}$ the direction of the normal of the cross-section A and $v_{n}$ the projection of the velocity vector on the normal. The Doppler velocity $v_{P W D}$ was converted to the angle-corrected velocity $v_{n}(t)$ as: $v_{n}(t)=\frac{v_{P W D}(t)}{\cos (\theta)}$, With $\theta$ the angle between the US beam and the assumed flow direction. In this study, $\theta$ was set to $70^{\circ}$.

However, evaluation of this surface integral is highly cumbersome using conventional PWD, since it typically entails a range of assumptions, which are potentially erroneous for complex flow conditions. Indeed, eq. 1 shows that a correct measurement of the flow $Q$ requires knowledge of the spatial velocity distribution in the considered cross-section. However, conventional PWD systems do not allow to simultaneously scan and process different sampling locations. Hence, we investigated two commonly applied strategies to circumvent a direct measurement of the flow profile:

(i) Large sample volume acquisition: Eq.1 is simplified to $Q=v_{n, \text { mean }}(t) * A$. with $v_{n, \text { mean }}$ the mean velocity in the considered cross-section, obtained by stretching the Doppler sample volume (SV) over the complete cross-section.

(ii) Small sample volume acquisition: The velocity is measured at a specific location of the cross-section (typically the centre point), and is further used to derive the spatial velocity profile by assuming the actual flow conditions. The flow $\mathrm{Q}$ is then obtained as: $Q=\beta * v_{n, \max }(t) * A$, with $\beta$ a correction factor that accounts for the shape of the velocity profile; $v_{n, \max }$ the velocity in the centre of the vessel crosssection and assumed to be the maximal velocity (as is the case for fully symmetrical flow). A flat $(\beta=1)$ or parabolic $(\beta=0.5)$ profile are often assumed, but we assumed more complex flow profiles using Womersley theory. This method was simulated by positioning a small sample volume $(\mathrm{SV}=0.39 \mathrm{~mm})$ in the centre of the cross-sectional area.

\section{RESUlTS}

\section{A. Simulated Doppler spectra}

Figure 2 shows the PWD-spectra when the small sample volume is positioned in the centre of the cross-section, for all 4 measuring locations (pre-operative: proximal and distal, post-operative: proximal and distal) (top-middle) and when a large sample volume covering the full cross-section was chosen (preoperative proximal). For the post-operative case, 


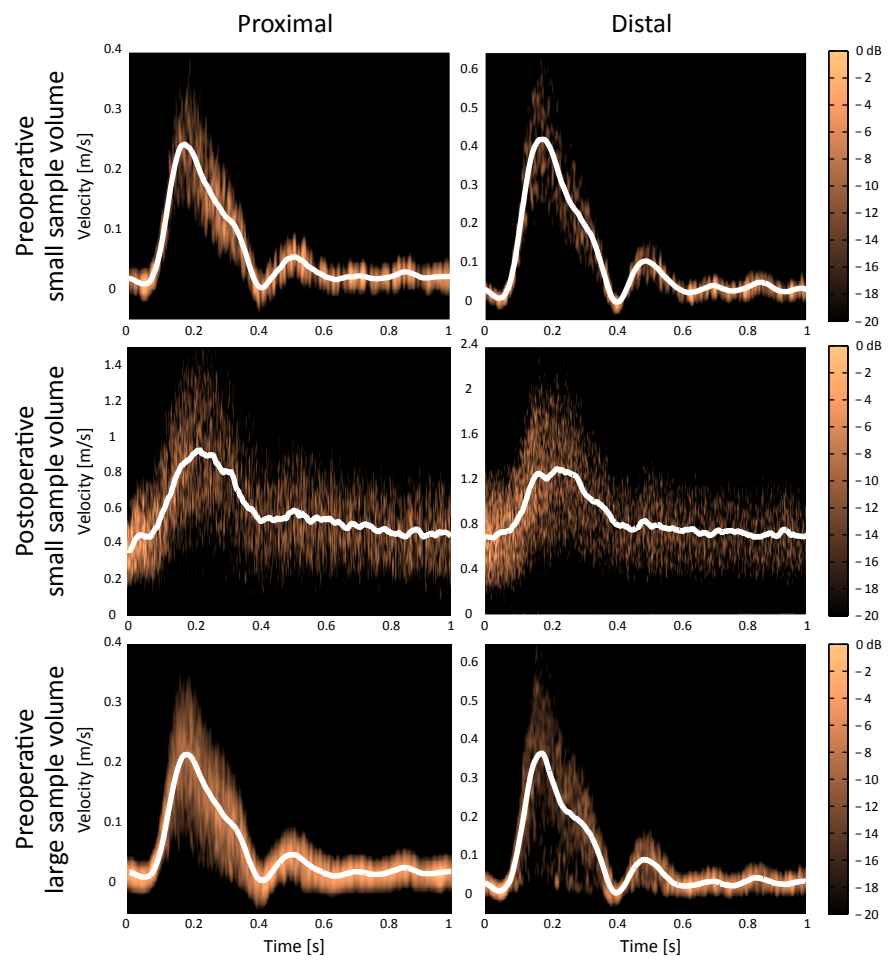

Fig. 2. Simulated PWD spectra for all 4 cases: pre-operative (proximal and distal), post-operative (proximal and distal). The lower panels show the large sample volume acquisitions for the pre-operative cases.

the complex flow conditions with high velocity magnitudes result in increased spectral broadening and less smooth velocity curves. For the large sample volume, the lower velocities near the vessel wall are also picked up, as can be observed for the spectra in the lower panels of fig.2.

\section{B. Velocity from PWD versus $C F D$}

We focus on the post-operative distal case (radial artery) when comparing the results from the synthetic PWD spectra to the true flow velocities (CFD), since the most complex flow
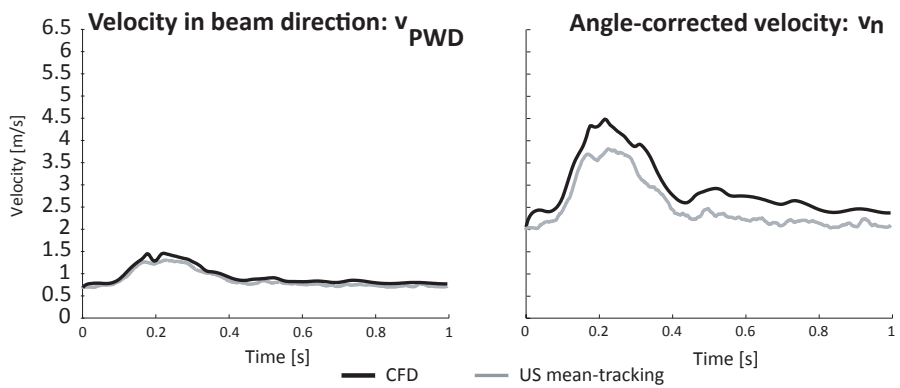

Fig. 3. The left panel shows the velocity as picked up by PWD (in the US beam direction), with the mean-tracking in solid grey and the CFD ground truth is displayed in solid black. The right panel shows the velocity after angle correction, with the same colour coding as the left panel. This comparison is illustrated for a small sample volume acquisition, in the post-operative distal region.
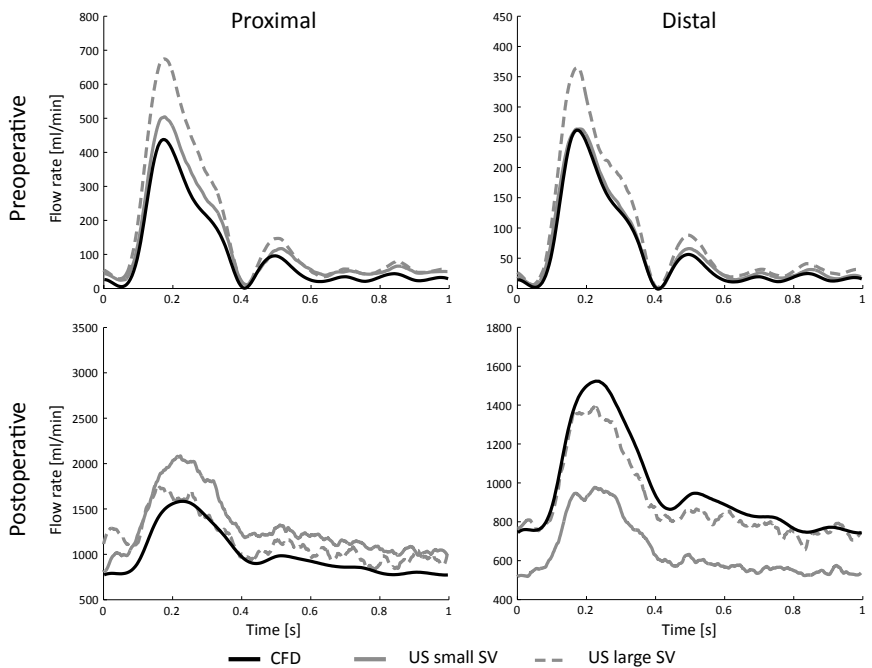

Fig. 4. Volume flows (solid grey), for a small sample volume (SV) acquisition, calculated with the $\beta$ value from Womersley theory are shown together with the volume flows (dashed grey) for large sample volume (SV) acquisition (equation 2) are compared to the CFD flow rate (solid black).

field was expected for this case. Figure 3 (left panel) compares the velocity $v_{P W D}$ as picked up by pulsed wave Doppler (in the direction of the ultrasonic beam), with the projection of the CFD velocity vector on this beam. Results are demonstrated for a small sample volume in the centre of the cross-section. Comparison with the ground truth shows a good agreement, with a slight underestimation of PWD (mean bias of $-8.1 \%$ ).

Figure 3 (right panel) compares the corresponding anglecorrected velocity $v_{n}$, with the projection of the CFD velocity vector on the normal of the cross-section. Angle correcting the mean tracking results in an underestimation (bias $=-14.2 \%$ ) post-operatively.

\section{Flow from $P W D$ versus $C F D$}

Figure 4 shows the volume flows obtained from the PWD spectra, for a small sample volume acquisition (solid grey for ultrasound), assuming a Womersley flow profile. The applied correction factors $\beta$ were $0.658,0.626,0.658$ and 0.627 for pre-operative proximal, pre-operative distal, post-operative proximal and post-operative distal respectively. While the proximal measurements (in the brachial artery) show a clear overestimation by ultrasound, results are less univocal for the distal site (radial artery).

TABLE I

THE BIAS FOR THE TIME-AVERAGE FLOW RATE

\begin{tabular}{|c|c|c|}
\hline Bias in $\%$ & Small sample volume & Large sample volume \\
\hline Preop prox & 30.1 & 67.6 \\
\hline Preop dist & 11.7 & 46.6 \\
\hline Postop prox & 29.8 & 14.2 \\
\hline Postop dist & -33 & -6.3 \\
\hline
\end{tabular}


The deviation between the simulated volume flow measurements and the ground truth is further quantified in table 1, providing an overview of the bias on the ultrasound data for the small and large sample volume acquisition. It is clear that the flow rates are overestimated by the US-algorithms, except for the post-operative distal case.

\section{DISCUSSION AND CONCLUSION}

In this paper, we studied PWD-based flow measurements in the brachial and radial artery in patients with an arteriovenous fistula, both in the pre- and post-operative case. We demonstrated that these PWD-based flow estimates are subject to a high degree of inaccuracy. This inaccuracy is not directly related to the inability of PWD to accurately capture the velocity component in the direction of the ultrasound beam, but is induced via two factors: (i) the angle-correction, which does not correctly estimate the velocity component along the assumed flow direction, and (ii) the lack of knowledge on the flow profile.

The inaccuracy induced by angle correction in volume flow estimation is demonstrated in fig.5. For uni-directional flow (fig.5, top), $v_{P W D}$ is the projection of the longitudinal velocity, $v_{x}$ (blue), which in this case equals the velocity vector $\vec{v}$. When a complex flow field is present (fig.5, bottom), $v_{P W D}$ not only consists of a contribution from the longitudinal component $v_{x}$ (blue), but also from the radial $v_{y}$ component (red). As such, the angle-corrected Doppler velocity $v_{n}(t)$ does not only reflect the longitudinal velocity component $v_{x}$ but
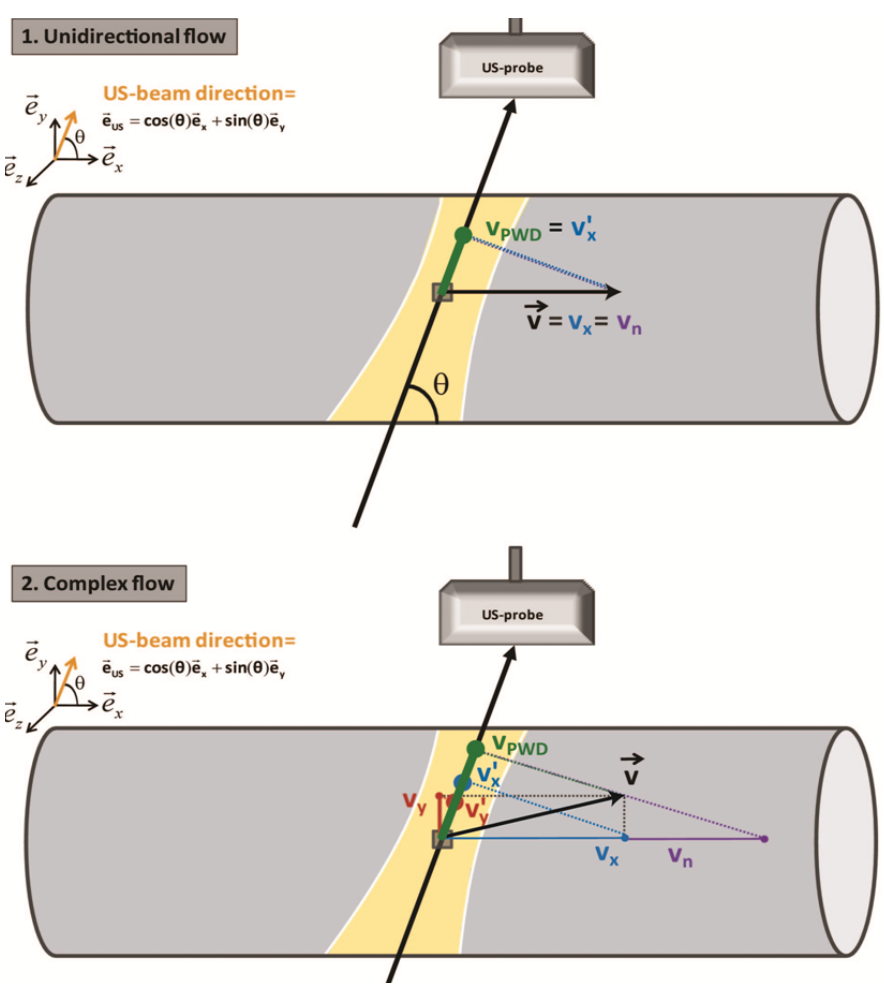

Fig. 5. Illustration on why angle correction of the Doppler velocity works for unidirectional flow (upper case 1) but fails for complex flow (lower case 2). also the radial velocity component $v_{y}$. Therefore, depending on the flow field and the orientation of the velocity vectors, angle-correction of $v_{P W D}$ can either over- or underestimate the velocity in the assumed flow direction $\left(v_{n}(t)\right)$.

Further, the lack of knowledge on the spatial velocity profile contributes to the inaccuracy of the flow estimation. When assessing the velocity in a sample volume positioned in the centre of the cross-section, a correction factor $\beta$ needs to be assumed, which relates to the shape of the velocity profile. Our data seem to indicate that, for the investigated cases, there is no single optimal choice for $\beta$. The $\beta$-values that one should use to match the PWD-derived velocity data, to the true mean velocity known from CFD are 0.506, 0.560, 0.507 and 0.935 for pre-operative proximal, pre-operative distal, postoperative proximal and post-operative distal, respectively. In the proximal locations, the assumption of a parabolic flow profile for a small centred sample volume $(\beta=0.5)$ seems reasonable. For the distal location in the small sample volume acquisition, the theoretical value ranges from 0.560 to 0.935 when changing from the pre- to the post-operative model.

When assuming fully symmetrical flow profiles, one expects that the use of the larger sample volume should provide the most accurate estimate of the volume flow. Interestingly, our data indicate that this was not systematically the case. First of all, this method is challenged since the velocities in the vessel wall region are difficult to measure, even in straight vessel segments. Furthermore, this method only assesses the mean velocity of the $1 \mathrm{D}$ velocity profile, instead of assessing the mean velocity for the complete $2 \mathrm{D}$ cross-section.

It is clear that, based on our study, it is difficult to provide strict guidelines to estimate blood flow rate by PWDacquisition. A potential solution for the demonstrated limitations regarding PWD volume flow measurements, is the use of 2D flow estimators (e.g. speckle tracking, vector Doppler), avoiding the need to angle correct a $1 \mathrm{D}$ velocity, but their clinical applicability is to be demonstrated. In parallel, researchers have focused on the development of multi-gate Doppler acquisitions, allowing to directly assess the velocity profile, through simultaneous processing of several sample volumes along the US beam.

\section{REFERENCES}

[1] T.C.Hodges, M. Fillinger, and R. Zwolak, "Longitudinal comparison of dialysis access methods: risk factors for failure," Journal of vascular surgery, vol. 26, pp. 1009-19, 1997

[2] D. Shemesh, I. Goldin, and D. Berelowitz, "Blood flow volume changes in the maturing arteriovenous access for hemodialysis.," Ultrasound in Medicine and Biology, vol. 33, pp. 727-733, 2007.

[3] A. Swillens, L. Lovstakken, J. Kips, H. Torp, and P. Segers, "Ultrasound simulation of complex flow velocity fields based on computational fluid dynamics," IEEE Transactions on Ultrasonics, Ferroelectrics and Frequency Control, vol. 56, no. 3, pp. 546-556, 2009.

[4] J. Jensen, "A new calculation procedure for spatial impulse responses in ultrasound," Journal of the acoustical society of America, vol. 105, pp. 3266-3274, 1999.

[5] J. A. Jensen, "Field: A program for simulating ultrasound systems," Medical and Biological Engineering and Computing, vol. 34, pp. 351352, 1996. 